Research report

\title{
The subiculum to entorhinal cortex projection is capable of sustaining both short- and long-term plastic changes ${ }^{\text {is }}$
}

\author{
Sarah Craig*, Sean Commins \\ Department of Psychology, National University of Ireland, Maynooth, Co. Kildare, Ireland \\ Received 13 April 2006; accepted 23 May 2006 \\ Available online 1 September 2006
}

\begin{abstract}
The hippocampus communicates with the neocortex via the entorhinal cortex. These areas are thought to be critically involved in the consolidation of memories. The hippocampus is considered to be the site of association of sensory information, which is then laid down for long-term storage in the neocortex. We examined the projection from the subiculum to the entorhinal cortex to determine whether it could function to transfer this hippocampally-processed information to the neocortex. Following stimulation in the subiculum we demonstrate a negative-going deflection followed by a positive-going deflection in the entorhinal cortex. This projection is capable of short-term plastic changes in the form of PPF. FIn addition, we demonstrate that long-term synaptic changes in the form of LTP and LTD could be sustained for at least 30 min on this pathway. Finally we show that PPF changes after LTP and LTD, suggesting that a presynaptic mechanism may be involved in both of these pathways.
\end{abstract}

(C) 2006 Elsevier B.V. All rights reserved.

Keywords: Long-term potentiation (LTP); Long-term depression (LTD); Paired-pulse facilitation (PPF); Hippocampus; Subiculum; Entorhinal cortex; Synaptic plasticity; Memory

\section{Introduction}

The hippocampal formation is considered to be critically involved in learning and memory [29,30,34,38]. Although it is believed to be the site of association of sensory information [35], allowing for rapid storage of this information, the hippocampus itself is not thought to be involved in the long-term storage of this information [39]. Instead long-term memory storage is thought to occur in the neocortex [25,37-39]. Therefore an interface between the hippocampus proper and the neocortex would be required to translate temporary hipppocampal information storage into a more permanent cortical storage.

The hippocampal formation is anatomically defined by a number of sub-regions that include the entorhinal cortex (EC) dentate gyrus (d.g.), areas CA3, CA1 and the subiculum. These structures are linked to each other by largely unidirectional projections. Entorhinal neurons constitute the major input to the hippocampus proper via the perforant path to the d.g. and is

\footnotetext{
is Grant Sponsor: Health Research Board of Ireland; Grant Number: HRB/RP/143/2002

* Corresponding author. Tel.: +353 1 7086069; fax: +353 17084767

E-mail address: Sarah.Craig@may.ie (S. Craig).
}

also considered to be the major recipient of its outputs. The subiculum and area CA1 are thought to constitute the major output structures of the hippocampus. The subiculum is the major synaptic relay for the majority of CA1 neurons $[1,40,41,45]$ making the subiculum the last synaptic relay of the hippocampal formation prior to the cortex. Thus, the subiculum could possibly act as such a mediator of hippocampal-cortical interaction as it also receives and integrates information from several different sources, such as the EC, peri- and postrhinal cortices and the presubiculum $[22,23,27,28,44,47]$. The subiculum can then pass this information to cortical regions either via the entorhinal cortex or by synapsing directly on perirhinal cortex (PER) or postrhinal cortex (POR) neurons [14,40].

The majority of back projections to the neocortex are mainly mediated through the EC [6] with anatomical studies $[43,46]$ suggesting the existence of a direct projection between the subiculum and the EC. However, the subiculum is not composed of a homogeneous cell population [42] and this has resulted in conflicting results with regards to its outputs. A study by Witter et al. [46] maintained that only the proximal part of the subiculum projects to the entorhinal cortex. An injection of PHA-L (anterograde tracer) in the distal subiculum did not result in labelling in the entorhinal cortex. However, later studies con- 
tradict this result. Tamamaki and Nojyo [43] used the tracer Dil and observed retrograde labelling in distal subiculum after injection in the medial entorhinal cortex (MEC). Based on this result these authors suggest that topography is maintained between the subiculum and EC. A recent study adds to the debate [21], and seems to confirm the earlier findings of Tamamaki and Nojyo [43]. Injections in the proximal dorsal subiculum with PHA-L and BDA resulted in a large amount of labelled cells in the lateral entorhinal cortex (LEC). Dye injection in the distal dorsal subiculum resulted in labelling of a small number of neurons in the most caudal part of MEC.

Current theories of hippocampal function in memory propose that connections between the hippocampus and neocortex undergo use-dependent changes in synaptic strength, enabling the consolidation of memory $[17,29,35]$. Paired-pulse facilitation (PPF) is a form of short-term synaptic plasticity. PPF is the phenomenon whereby the field excitatory postsynaptic response (fEPSP) to a second stimulus is enhanced relative to the first, if the second stimulus is delivered relatively quickly after the first [50]. PPF is thought to be primarily a presynaptic phenomenon, resulting from a transient increase in calcium levels in the presynaptic terminal caused by the first stimulus elevating the response to the second stimulus [48]. Long-term potentiation (LTP) and depression (LTD) have been considered as possible models for the synaptic changes that occur during learning and memory in the CNS $[3,4,18]$. LTP is a long-lasting increase in monosynaptic responsiveness induced by short high-frequency activation of the appropriate monosynaptic inputs [4]. In contrast LTD of synaptic transmission is the persistent decrease in synaptic response as a result of repeated low-frequency stimulation (LFS) of the pathway. It is a matter of controversy whether LTP/LTD is primarily a presynaptic or a postsynaptic phenomenon or some combination of the two [3]. It is hypothesised that if LTP, or LTD expression includes a presynaptic locus, then it might alter expression of PPF $[8,20]$. LTP, which increases synaptic efficacy, causes a reduction in PPF, post-LTP induction [9,36]. Commins et al. [9], for example have reported that PPF decreased after LTP in the projection from CA1 to the subiculum and in the CA1 to prefrontal cortex projection PPF both increased and decreased post-LTP [36]. On the other hand LTD, which decreases synaptic efficacy [15] should cause an increase in facilitation post-LTD induction. Commins and O'Mara [12] observed such an increase in PPF 30 min post-LFS in the projection between the area CA1 and the subiculum.

In this study we first investigate whether there is physiological evidence for a projection from the subiculum to the entorhinal cortex. We determine whether this projection is capable of sustaining both short-term (in the form of PPF) and long-term plasticity (in the form of LTP/LTD). Finally we investigate the interaction effect between these forms of synaptic changes.

\section{Materials and methods}

\subsection{Subjects and surgery}

Adult male Wistar rats (weight 200-300 g) were anaesthetized with urethane (ethyl carbamate: $1.5 \mathrm{~g} / \mathrm{kg}$, i.p.) and mounted on a stereotaxic holder. A local anaesthetic/adrenaline combination was injected under the scalp and an incision was made to visualize the skull. Burrholes for the stimulating and recording electrodes (stainless bipolar wires insulated except at tips; $50 \mu \mathrm{m}$ ) were placed at the coordinates relative to Bregma [33]: stimulating electrodes aimed at the subiculum: $6.3 \mathrm{~mm}$ posterior and $4.4 \mathrm{~mm}$ lateral; recording electrodes, aimed at EC: $6.7 \mathrm{~mm}$ posterior and $5.8 \mathrm{~mm}$ lateral. Signals were filtered between $0.1 \mathrm{~Hz}$ and $1 \mathrm{kHz}$ and then amplified. Recordings were digitised online using a PC connected to a CED-1401 plus interface (CED, Cambridge, UK).

\subsection{Procedures}

The depth profile of the subiculum to entorhinal cortex projection was examined by firstly slowly lowering the recording electrode towards the EC and allowing it settle for a minimum of $10 \mathrm{~min}$. The stimulating electrode was slowly lowered in $50 \mu \mathrm{m}$ steps towards the dorsal subiculum. The final depths of both electrodes were adjusted until the maximal fEPSP was recorded. At each of the $50 \mu \mathrm{m}$ steps 2 stimuli of $1 \mathrm{~mA}$ were given at a low repetition rate (interpulse interval of $20 \mathrm{~s}$ ). The first stimulus was used as a test stimulus and only the second was subsequently analysed.

Baseline PPF effects were measured after allowing both the stimulating and recording elecrodes to settle for $10 \mathrm{~min}$ in the subiculum and the entorhinal cortex, respectively. Pairs of stimuli were then delivered with inter-stimulus intervals (ISIs) of 20, 40, 60, 120, 240, and $480 \mathrm{~ms}$. The first fEPSP and second fEPSP elicited by the first and second stimulus of the stimulus pair will be referred to as fEPSP1 and fEPSP2, respectively. The PPF value was calculated by taking the average of six slope values of fEPSP1, for a given ISI, and normalizing the average of six values for fEPSP2 with respect to this value in percentage terms (see [9] for further details).

The same protocol for PPF was used pre- and $30 \mathrm{~min}$ post-LTP/LTD induction (see below).

Induction of LTP was attempted using a high-frequency stimulation (HFS) protocol, following a minimum of $10 \mathrm{~min}$ baseline period at a low frequency stimulation rate (LFS $0.5 \mathrm{~Hz}$ ). HFS consisted of 3 trains of stimuli for $200 \mathrm{~ms}$ at $250 \mathrm{~Hz}$, with an intertrain interval of $30 \mathrm{~s} \mathrm{[16].} \mathrm{Stimulus} \mathrm{intensity} \mathrm{during} \mathrm{LTP}$ induction was set at baseline intensity. Following this, low-frequency stimulation was then resumed at a rate of $0.5 \mathrm{~Hz}$ for a further $30 \mathrm{~min}$.

Induction of LTD was attempted using a low-frequency stimulation (LFS) protocol, following a $10 \mathrm{~min}$ baseline period as above. LFS consisted of 900 stimuli at $1 \mathrm{~Hz}$ (see [2] for details). Stimulus intensity during LTD induction was set at baseline intensity. Low-frequency stimulation was then resumed at a rate of $0.5 \mathrm{~Hz}$ for a further $30 \mathrm{~min}$.

\subsection{Histological processing}

After all experiments the rats were overdosed with sodium pentobarbitone and their brains subsequently removed. In order to check the positions of the electrodes the brains were frozen in Tissue-Tek O.C.T. compound (R.A. Lamb, East Sussex, UK). Twenty-micrometer coronal slices were cut using a cryostat. Sections with electrode lesions were mounted on slides in order to confirm electrode positioning. The sections were counterstained with cresyl violet (Nissl) to defind cytoarchitectonic borders and subsequently coverslipped. All electrode sites and electrode tracks were reconstructed using Paxinos and Watson altas [33].

\subsection{Statistical analysis}

A series of independent $t$-tests and one-way analyses of variance with the appropriate post hoc test (Tukey at the 5\% level of significance) were used; ${ }^{*} p<0.05,{ }^{* *} p<0.01,{ }^{* * *} p<0.001$.

\section{Results}

\subsection{Depth profile of projection from subiculum to entorhinal cortex}

In a first set of experiments we wished to examine the depth profile of the projection from the dorsal subiculum to EC. In 
addition, we wished to describe the characteristic response of the entorhinal neurons to subicular stimulation. In an initial set of experiments (data not shown) we lowered the recording electrode into more medial regions of EC and then slowly lowered the stimulating electrode into more distal regions of the subiculum. Unfortunately we were unable to evoke a strong response that would enable us to characterise this projection. We then lowered the recording electrode into more laterally-placed entorhinal neurons, while slowly lowering the stimulating electrode into more proximally placed regions of the subiculum. The following results describe responses obtained mainly on the proximal subiculum to lateral entorhinal projection. Fig. 1a shows representative Nissl stained slices with a typical stimulating and recording electrode in the subiculum and EC, respectively.

In all cases $(n=6)$ a response was evoked in the EC following stimulation in the dorsal subiculum. The stimulating sites were positioned in the dorsal subiculum, located just below the corpus callosum. The positions of the stimulating electrodes were all located between 6.1 and $6.4 \mathrm{~mm}$ posterior to Bregma. In addition the final positions of the recording electrodes in LEC were all located between 6.7 and $7.2 \mathrm{~mm}$ posterior to Bregma.

The stimulating electrode was slowly lowered toward the dorsal subiculum (Fig. 1b). The recording electrode was lowered $6 \mathrm{~mm}$ below the surface of the brain and allowed to settle in the EC (Fig. 1b). Stimulation (at a rate of $0.05 \mathrm{~Hz}$ ) of different sites en route to the subiculum evoked responses in the EC. In all cases the amplitude and slope of the evoked response increased as the electrodes were lowered toward the dorsal subiculum.

No response was observed in EC as the stimulating electrode was lowered through the occipital cortex (Fig. 1c, 1-3). As the electrode was lowered toward the subiculum a positive-going deflection was first observed in EC at the border of the occipital cortex and the corpus callosum (see Fig. 1c, 4). At the border of the corpus callosum and the subiculum the latency of the

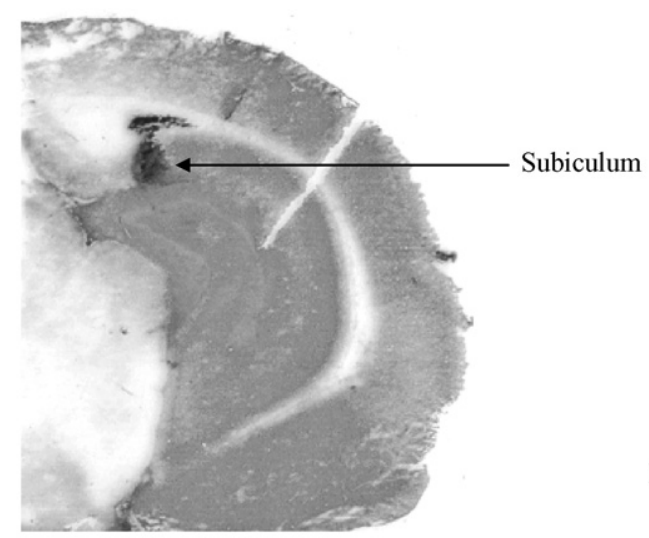

(a)
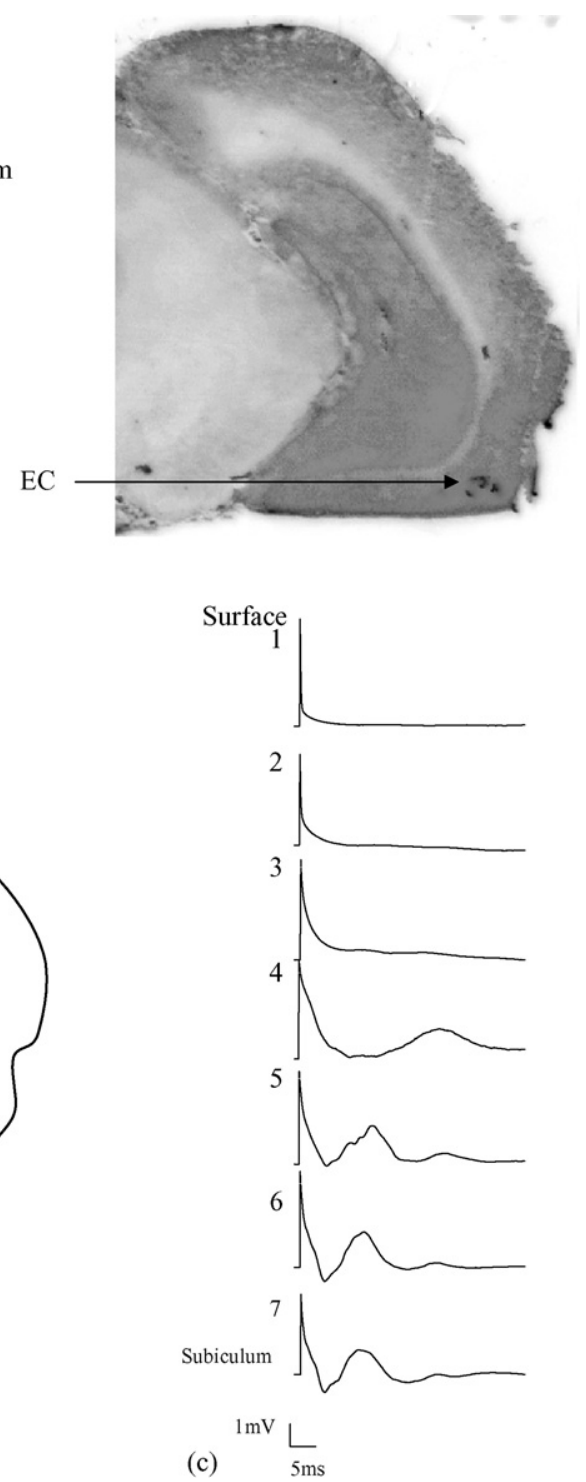

Fig. 1. (a) Positions of the recording electrode in EC and of the stimulating electrodes at seven representative positions en route to subiculum; (b) representative depth profile of projection from subiculum to EC, showing sample EPSP responses at seven representative positions (1-7) from surface to subiculum; (c) Nissl-stained coronal sections, showing electrode tracks of subiculum and tracks of EC. 
positive wave decreased and was preceded by a negative-going deflection (Fig. 1c, 5 and 6). This response increased as the electrode approached the subiculum. A single-pulse stimulation in the dorsal subiculum evoked a negative-going deflection followed by a positive-going deflection in the EC (see Fig. 1c, 7). This negative-going deflection occurred at $6.85 \pm 0.9 \mathrm{~ms}$ and had a mean peak amplitude of $-1.46 \pm 0.13 \mathrm{mV}$. The positive deflection occurred later at a mean latency value of $14.84 \pm 0.88 \mathrm{~ms}$ and had a mean peak value of $2.13 \pm 0.22 \mathrm{mV}$ and a slope of $0.8 \pm 0.14 \mathrm{mV} / \mathrm{ms}$ (defined in terms of the ascending component of the positive-going deflection).

\subsection{Experiment 2}

In a second set of experiments we wished to determine whether the subiculum to EC projection was capable of sustaining both short- and long-term plastic effects (measured in terms of PPF and LTP). In addition we wished to determine whether there was an interaction between these two forms of plasticity. After allowing both stimulating and recording electrodes to settle in the subiculum and EC, respectively, for $10 \mathrm{~min}$ PPF was measured at different ISIs six times for five animals, giving a minimum of 30 measurements for each interval tested. A strong PPF effect was evident with PPF appearing at the first stimulus interval $(20 \mathrm{~ms}$, fEPSP2 PPF value of $166.8 \pm 9.5 \%$ the value of fEPSP1) and was still evident at $480 \mathrm{~ms}$ (Fig. 2a). A repeated-measures ANOVA confirmed there was an overall PPF effect within groups $(F=22.327$; d.f. $=1,24 ; p<0.001)$. Subsequent $t$-tests found that all paired comparisons of interest were significantly different from each other $(p<0.001)$.

Following PPF we again allowed electrodes to settle for a minimum of $10 \mathrm{~min}$ while recording baseline responses. LTP was then attempted using a HFS protocol to determine whether this projection was capable of sustaining long-term plastic effects. Fig. 2b demonstrates that LTP was successfully induced and that evoked responses remained potentiated for at least $30 \mathrm{~min}$. A one-way ANOVA was conducted to compare the $10 \mathrm{~min}$ baseline period before HFS with the $0-10$ and 20-30 min periods post-HFS for mean slope responses. The overall analyses of variance was statistically significant $(F=264.1$; d.f. $=2,71 ; p<0.001)$. Subsequent post hoc comparisons (Tukey, $p<0.05$ ) revealed that the mean response during the $0-10 \mathrm{~min}(131 \pm 1.2 \%$ of baseline $)$ and the $20-30 \mathrm{~min}$ periods post-HFS $(131.83 \pm 1.08 \%$ of baseline) were significantly higher than the $10 \mathrm{~min}$ baseline period.

At 30 min post-HFS, PPF was measured for the second time to determine any interaction effects between LTP and PPF. An overall general decrease in PPF was observed at 30 min post-LTP (see Fig. 2c). Analysis revealed that there was a significant decrease in percentage facilitation post-HFS compared to baseline facilitation values. This reduction was significant at the following intervals: 20, 120, 240 and $480 \mathrm{~ms}(p<0.01)$.

\subsection{Experiment 3}

In a third set of experiments we wished to determine whether the subiculum to EC projection was capable of sustaining short and long-term plastic effects, in terms of PPF and LTD. We also wished to examine the interaction between these two forms of plasticity in this pathway. After allowing stimulating and recording electrodes to settle in the subiculum and EC, respectively, PPF was measured at different ISIs six times for a further five animals, giving a minimum of 30 measurements for each interval tested. Similar to above a strong baseline PPF effect was evident with PPF appearing at the first stimulus interval (20 ms, fEPSP2 PPF value of $167.6 \pm 8.4 \%$ the value of fEPSP1) and was still evident at $480 \mathrm{~ms}$ (Fig. 3a). A repeated-measures ANOVA again confirmed there was an overall PPF effect within groups $(F=88.212$; d.f. $=1,24 ; p<0.001)$. Subsequent $t$-tests found that all paired comparisons of interest were significantly different from each other $(p<0.001)$.

Following PPF, we again allowed to electrodes to settle for a minimum of 10 min while recording baseline responses. To determine whether the projection from the subiculum to EC was capable of sustaining LTD, a low frequency protocol was used. Fig. $3 b$ demonstrates that LTD was successfully induced and that evoked responses remained depressed for at least $30 \mathrm{~min}$. A one-way ANOVA was conducted to compare the $10 \mathrm{~min}$ baseline period before LTD induction with the $0-10$ and $20-30$ min periods post-LFS for slope measures. The overall analyses of variance was statistically significant $(F=168.4 ;$ d.f. $=2,71 ; p<0.001)$. Subsequent post hoc comparisons (Tukey, $p<0.05$ ) revealed that the mean response during the $0-10 \mathrm{~min}(74.7 \pm 0.9 \%$ of baseline $)$ and the $20-30 \mathrm{~min}$ periods post-LFS $(85.71 \pm 0.98 \%$ of baseline) were significantly lower than the $10 \mathrm{~min}$ baseline period.

Finally we wished to determine if there was an interaction effect between PPF and LTD. At $30 \mathrm{~min}$ post-LTD induction, PPF was again examined across the range of ISIs used previously. A significant increase was observed in the percentage facilitation post-LFS compared to the baseline facilitation at the ISI of $20 \mathrm{~ms}(p<0.05)$. However, significant decreases in facilitation were observed at the 120, 240 and 480 ms ISIs $(p<0.05)$, while no change in facilitation was noted for the remaining intervals $(40,60 \mathrm{~ms})$.

\section{Discussion}

It has been proposed that connections between the hippocampus and the neocortex undergo use-dependent synaptic changes, enabling the consolidation of memories [17,29,35]. Rolls [35] suggests that the hippocampus operates as an intermediate-term memory and that long-term memories are laid down in the neocortex. In addition Rolls suggests that all back projections from the hippocampus to the neocortex should be modifiable. This is in contrast with McClelland et al. [25] who suggests that CA3-CA1 projection is the last set of synapses to be modifiable. Nadel and Moscovitch [29] proposed that the interaction between the hippocampus and the neocortex itself is the memory trace. However most theories recognise the need for an interface between the presumed memory function of the hippocampus and the neocortex.

The hippocampal output to the neocortex is mainly mediated through the EC [6], with the subiculum to EC projection being 

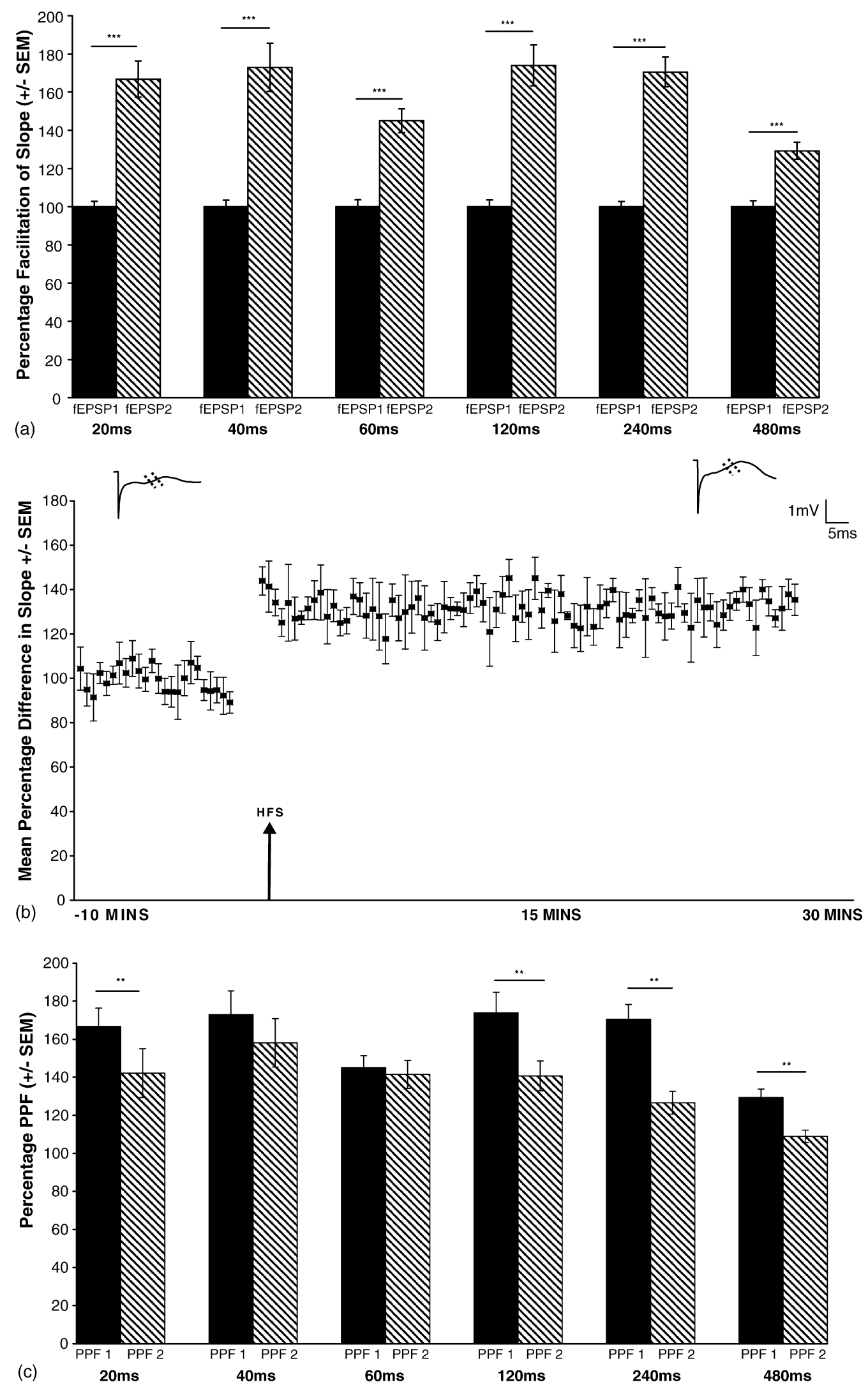

Fig. 2. (a) A bar chart showing paired-pulse facilitation in the subiculum-EC pathway before LTP induction for intervals indicated; bars represent mean peak heights for fEPSP1 (black) and fEPSP2 (hatched). Data are normalised to fEPSP1 (100\%); (b) effects of HFS on the slope of fEPSPs. The post-HFS values are expressed as percentage of the pre-stimulation baseline \pm S.E.M. Representative traces for pre- and post-HFS above graph; (c) a bar chart showing changes in paired-pulse facilitation after LTP was induced. Bars represent mean PPF before (black) and after (hatched) HFS that induced LTP. 

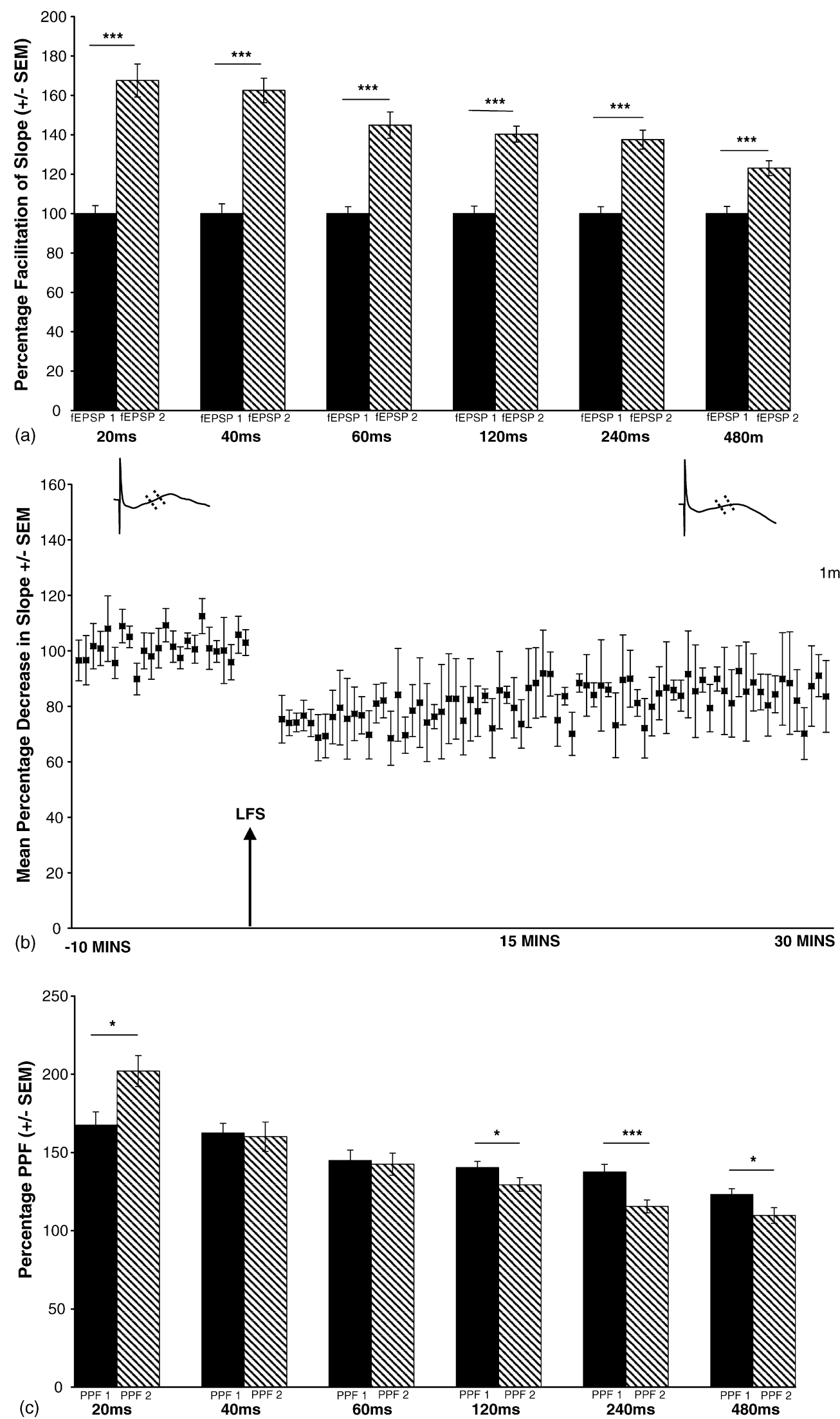

Fig. 3. (a) A bar chart showing paired-pulse facilitation in the subiculum-EC pathway before LTD induction for intervals indicated; bars represent mean peak heights for fEPSP1 (black) and fEPSP2 (hatched). Data are normalised to fEPSP1 (100\%); (b) effects of LFS on the slope of fEPSPs. The post-LFS values are expressed as percentage of the pre-stimulation baseline \pm S.E.M. Representative traces for pre- and post-LFS above graph; (c) a bar chart showing changes in paired-pulse facilitation after LTD was induced. Bars represent mean PPF before (black) and after (hatched) LFS that induced LTD. 
the final relay, which would function to transfer hippocampallyprocessed information to the neocortex for storage. There is conflicting information regarding the nature of subiculum to EC projection (see Section 1) but most authors agree that this projection is topographical in nature. Although we were unable to obtain a strong physiological response of the distal subiculum to MEC, we did find the proximal to LEC projection produced a strong response characterised by a negative-going deflection followed by a positive-going deflection.

Although we cannot state for certain. We further suggest that this projection may be monosynaptic in nature. Several previous studies have interpreted a short latency as consistent with monosynaptic activation $[5,13]$. Consistent with this, we have observed similar short EPSP peak latencies in the subiculum to EC projection. Other authors [49] suggest that if the components of the EPSP remain intact following trains of continuous stimulation, that this demonstrates evidence for a monosynaptic response, although the frequency of stimulation varies (from $100 \mathrm{~Hz}$ according to Cousens and Otto [13], to $>25 \mathrm{~Hz}$ according to others [24,49]). In this study, components of the fEPSP response in the EC following subiculum stimulation at both 1 and $250 \mathrm{~Hz}$ remained intact. Again this suggests that this projection may indeed be monosynaptic in nature.

We provide evidence here for the first time, that synaptic plasticity exists in the projections from the subiculum to EC. We were able to demonstrate that this pathway was capable of PPF across a range of intervals (similar to that seen on the CA1 to subiculum pathway [9]). In addition we were able to induce long-term changes in this pathway that lasted for at least $30 \mathrm{~min}$. These changes were in the form of both LTP and LTD, giving credence to the suggestion by Rolls [35] that the back projections from the hippocampus to the EC should be capable of undergoing synaptic plasticity. LTP has also been observed on the CA1-subiculum projection $[10,11]$, the CA1 to EC projection (unpublished observations) and now on the subiculum to EC projection. However, in contrast to LTP, LTD has been difficult to demonstrate in vivo. Using a variety of stimulation protocols, Anderson et al. [2] were unable, for example, to induce LTD on the CA1 to subiculum pathway. However, in this study we were able to induce LTD in the projection from subiculum to $\mathrm{EC}$, which was sustained for at least $30 \mathrm{~min}$. There is evidence to suggest that LTP and LTD may be dependent on distinct and separate mechanisms $[7,19,31,32]$. However both are thought to form the basis of memory formation and storage. The distinct roles of either LTP or LTD have yet to be elucidated. And why one set of projection should sustain LTD while another does not is still uncertain. It is possible that bi-directional plasticity is necessary for memory consolidation and transfer from the hippocampal neurons to the neocortex.

Whether LTP/LTD has a pre- or post-synaptic locus is a matter of debate. To help better understand this, McNaughton [26] developed an approach based on the analysis of changes in PPF following LTP. These authors suggest that PPF should change following LTP if presynaptic mechanisms contribute to LTP expression. In this study we found that PPF decreased after LTP compared to the baseline PPF measurements. These results support the hypothesis that LTP expression includes a presynaptic locus. In contrast, PPF in the hippocampus has been reported to increase post-LFS [12], if it includes a presynaptic component (see Section 1). In this study we observed an increase in PPF post-LTD for the $20 \mathrm{~ms}$ interval only. In addition, PPF decreased at the 120, 240 and $480 \mathrm{~ms}$ intervals. Thus, we conclude that in this projection, LTD expression is not only associated with a presynaptic change, but with some other mechanism.

To conclude, in this study we confirmed the presence of a projection from the subiculum to the entorhinal cortex. This pathway was capable of undergoing both short- and long-term synaptic changes. This suggests that the subiculum may act as a possible interface between the hippocampus and the neocortex during the consolidation of memories. We also suggest that the expression of LTP includes a presynaptic locus but cannot be certain about the mechanism involved in LTD induction in this pathway.

\section{Acknowledgements}

The authors gratefully acknowledge the help of Shane O'Mara and Thelma Crowley (Trinity College) and Derek Walsh (NUI Maynooth).

\section{References}

[1] Amaral DG, Dolorous C, Alvarez-Roy P. Organisation of CA1 projections to the subiculum: a PHA-L analysis in the rat. Hippocampus 1991;1:415-36.

[2] Anderson M, Commins S, O'Mara SM. The effects of low-frequency and two-pulse stimulation protocols on synaptic transmission in the CA1subiculum pathway in the anaesthetised rat. Neurosci Lett 2000;279:181-4.

[3] Bliss TVP, Lomo T. Long-lasting potentiation of synaptic transmission in the dentate area of the anaesthetized rabbit following stimulation of the perforant path. J Physiol (London) 1973;232:331-56.

[4] Bliss TVP, Collingridge GL. A synaptic model for memory: long-term potentiation in the hippocampus. Nature 1993;361:31-9.

[5] Burette F, Jay TM, Laroche S. Reversal of LTP in the hippocampal afferent fiber system to the prefrontal cortex in vivo with low-frequency patterns of stimulation that do not produce LTD. J Neurophysiol 1997;78:1155-60.

[6] Burwell RD, Amaral DG. Cortical afferents of the perirhinal, postrhinal and entorhinal cortices of the rat. J Comp Neurol 1998;398:179-205.

[7] Chabot C, Gagne J, Giguere C, Bernard J, Baury M, Massicotte G. Bidirectional modulation of AMPA receptor properties by exogenous phospholipase $A_{2}$ in the hippocampus. Hippocampus 1998;8:299-309.

[8] Christie BR, Abraham WC. Differential regulation of paired-pulse plasticity following LTP in the dentate gyrus. Neuroreport 1994;5:385-8.

[9] Commins S, Gigg J, Anderson M, O’Mara SM. Interaction between pairedpulse facilitation and long-term potentiation in the projection from hippocampal area CA1 to the subiculum. Neuroreport 1998;9:4109-13.

[10] Commins S, Gigg J, Anderson M, O’Mara SM. The projection from hippocampal area CA1 to the subiculum sustains long-term potentiation. Neuroreport 1998;9:847-50.

[11] Commins S, Aggleton JP, O'Mara SM. Physiological evidence for a possible projection from dorsal subiculum to hippocampal area CA1. Exp Brain Res 2002;146:155-60.

[12] Commins S, O’Mara SM. Interactions between paired-pulse facilitation, low-frequency stimulation, and behavioural stress in the pathway from hippocampal area CA1 to the subiculum: dissociation of baseline synaptic transmission from paired-pulse facilitation and depression of the same pathway. Psychobiology 2000;28:1-11.

[13] Cousens G, Otto TA. Induction and transient suppression of long-term potentiation in the peri- and postrhinal cortices following theta-related stimulation of hippocampal field CA1. Brain Res 1998;780:95-101. 
[14] Deacon TW, Eichenbaum H, Rosenberg P, Eckmann KW. Afferent connections of the perirhinal cortex in the rat. J Comp Neurol 1983;220: 168-90.

[15] Dudek SM, Bear MF. Homosynaptic long-term depression in area CA1 of hippocampus and effects of $N$-methyl-D-aspartate receptor blockade. Proc Natl Acad Sci 1992;89:4363-7.

[16] Gooney M, Shaw K, Kelly A, Lynch MA. Long-term potentiation and spatial learning are associated with increased phosphorylation of TrkB and extracellular signal-regulated kinase (ERK) in the dentate gyrus: evidence for a role for brain-derived neurotrophic factor. Behav Neurosci 2002;116:455-63.

[17] Graham KS, Hodges JR. Differentiating the roles of the hippocampal complex and the neocortex in long-term storage: evidence from the study of semantic dementia and AD. Neuropsychology 1997;11:1-13.

[18] Heynen AJ, Abraham WC, Bear MF. Bidirectional modification of CA1 synapses in the adult hippocampus in vivo. Nature 1996;381: 163-6.

[19] Hrabetova S, Sacktor TC. Bidirectional regulation of protein kinase Mzeta in the maintenance of long-term potentiation and long-term depression. $\mathrm{J}$ Neurosci 1996;16:5324-33.

[20] Kleschevnikov AM, Sokolov MV, Kuhnt U, Dawe GS, Stephenson JD, Voronin LL. Changes in paired-pulse facilitation correlate with induction of long-term potentiation in area CA1 of rat hippocampal slices. Neuroscience 1997;76:829-43.

[21] Kloosterman F, Witter MP, van Haeften T. Topographical and laminar organization of subicular projections to the parahippocampal region of the rat. J Comp Neurol 2003;455:156-71.

[22] Kohler C. Intrinsic connections of the retrohippocampal region in the rat brain. The subicular complex. J Comp Neurol 1985;236:504-22.

[23] Kosel KC, Van Hoesen GW, Rosene DL. A direct projection from the perirhinal cortex (area 35) to the subiculum in the rat. Brain Res 1983;269:347-51.

[24] Leung LS. Orthodromic activation of hippocampal CA1 region of the rat. Brain Res 1979;176:49-63.

[25] McClelland JL, Mc Naughton BL, O'Reilly RC. Why are there complementary learning systems in the hippocampus and neocortex: insights from the successes and failures of connectionist models of learning and memory. Psychol Rev 1995;102:419-57.

[26] McNaughton BL. Long-term synaptic enhancement and short-term potentiation in rat fascia dentata act through different mechanisms. J Physiol (London) 1982;324:249-62.

[27] Naber PA, Witter MP, Lopes da Silva FH. Perirhinal cortex input to the hippocampus in the rat: evidence for parallel pathways, both direct and indirect. A combined physiological and anatomical study. Eur J Neurosci 1999;11:4119-33.

[28] Naber PA, Witter MP, Lopes da Silva FH. Evidence for a direct projection from postrhinal cortex to the subiculum in the rat. Hippocampus 2001;11:105-17.

[29] Nadel L, Moscovitch M. Memory consolidation, retrograde amnesia and the hippocampal complex. Curr Opin Neurobiol 1997;7:217-27.

[30] O'Keefe J, Nadel L. The hippocampus as a cognitive map. Oxford: The Clarendon Press; 1978.

[31] Oliet SHR, Malenka RC, Nicoll RA. Bidirectional control of quantal size by synaptic activity in the hippocampus. Science 1996;271:1294-7.
[32] Palmer MJ, Irving AJ, Seabrook GR, Jane DE, Collingridge GL. The group I mGlu receptor agonist DHPG induces a novel form of LTD in the CA1 region of the hippocampus. Neuropharmacology 1997;36:1517-32.

[33] Paxinos G, Watson C. The rat brain in stereotaxic coordinates. San Diego: Academic Press; 1998.

[34] Rolls ET, O'Mara SM. Neurophysiological and theoretical analysis of how the primate hippocampus functions in memory. In: Ono T, Squire LR, Raiche ME, Perrett DI, Fukuda M, editors. Brain mechanisms of perception: from neuron to behaviour. New York: Oxford University Press; 1993. p. $276-300$

[35] Rolls ET. A theory of hippocampal function in memory. Hippocampus 1996;6:601-20.

[36] Schulz PE, Cook EP, Johnston D. Changes in paired-pulse facilitation suggest presynaptic involvement in long-term potentiation. J Neurosci 1994;14:5325-37.

[37] Squire LR, Cohen NJ, Nadel L. The medial temporal regions and memory consolidation: a new hypothesis. In: Weingartner $\mathrm{H}$, Parker E, editors. Memory consolidation. Hillsdale, NJ: Erlbaum; 1984. p. 185-210.

[38] Squire LR. Memory and the hippocampus: a synthesis from findings with rats, monkeys, and humans. Psychol Rev 1992;99:195-231.

[39] Squire LR, Alvarez P. Retrograde amnesia and memory consolidation: a neurobiological perspective. Curr Opin Neurobiol 1995;5:178-83.

[40] Swanson LW, Cowan WM. An autoradiographic study of the organization of the efferent connections of the hippocampal formation in the rat. J Comp Neurol 1977;172:49-84.

[41] Swanson LW, Wyss JM, Cowan WM. An autoradiographic study of the organisation of intrahippocampal association pathways in the rat. J Comp Neurol 1978;181:681-716.

[42] Swanson LW, Sawchenko PE, Cowan WM. Evidence for collateral projections by neurons in Ammon's horn, the dentate gyrus and subiculum: a multiple retrograde labelling study in the rat. J Neurosci 1981;1:548-59.

[43] Tamamaki N, Nojyo Y. Preservation of topography in the connections between the subiculum, field CA1 and the entorhinal cortex in rats. J Comp Neurol 1995;353:379-90.

[44] Witter MP. Connectivity of the rat hippocampus. In: Chan-Palay V, BenAri Y, editors. The hippocampus-new vistas: neurology and neurobiology, vol. X. New York: Alan R. Liss Inc.; 1989. p. 67-82.

[45] Witter MP. Organization of the entorhinal-hippocampal system: a review of current anatomical data. Hippocampus 1993;3:33-44.

[46] Witter MP, Ostendorf RD, Groenewegen HJ. Heterogeneity in the dorsal subiculum of the rat. Distinct neuronal zones project to different cortical and subcortical targets. Eur J Neurosci 1990;2:718-25.

[47] Witter MP, Naber PA, van Haeften T, Machielsen WCM, Rombouts SARB Barkhof F, Scheltens P, Lopes da Silva FH. Cortico-hippocampal communication by way of parallel parahippocampal-subicular pathways. Hippocampus 2000;10:398-410.

[48] Wu LG, Saggau P. Presynaptic calcium is increased during normal synaptic transmission and paired-pulse facilitation, but not in long-term potentiation in area CA1 of hippocampus. J Neurosci 1994;14:645-54.

[49] Yeckel MF, Berger TW. Feedforward excitation of the hippocampus by afferents from the entorhinal cortex: redefination of the role of the trisynaptic pathway. Proc Natl Acad Sci 1990;87:5832-6.

[50] Zucker RS. Short-term synaptic plasticity. Annu Rev Neurosci 1989;12: 13-31. 